\title{
Domain of definition of Levermore's five-moment system
}

\author{
Michael Junk \\ Institut für Techno- \\ und Wirtschaftsmathematik \\ Kaiserslautern, Germany
}

\begin{abstract}
The simplest system in Levermore's moment hierarchy involving moments higher than second order is the five-moment closure. It is obtained by taking velocity moments of the one-dimensional Boltzmann equation under the assumption that the velocity distribution is a maximum-entropy function. The moment vectors for which a maximum-entropy function exist consequently make up the domain of definition of the system. The aim of this article is a complete characterization of the structure of the domain of definition and the connected maximum-entropy problem. The space homogeneous case of the equation and numerical aspects are also addressed.
\end{abstract}

Key words: Levermore's moment closure; maximum-entropy; moment realizability; reduced Hamburger moment problem

\section{Introduction}

Classically, the Euler equations are used to describe the time-evolution of a gas which is close to local thermodynamical equilibrium. In this macroscopic theory the state of the gas is characterized by quantities such as mass, momentum and energy. In cases of considerable deviation from equilibrium one might expect that more and more variables are required to describe the state of the gas. This vague idea has been made precise in the work by Levermore [6]. Based on the kinetic theory of gases he derives a whole hierarchy of relaxation systems with the Euler equation as first member. The approach, which 
can also be generalized to other kinetic theories, provides the systems with remarkable properties.

In the kinetic theory, a gas composed of identical particles is described by the time-evolution of a nonnegative distribution function $f(x, v, t)$ specifying the density of particles with velocity $v$ at time $t$ and position $x$. For simplicity, we will consider a hypothetical, one-dimensional gas where the particle velocities are scalars. Correspondingly, position space will also be one-dimensional in the following.

The evolution of the distribution function is usually governed by a kinetic equation like the Boltzmann equation

$$
\partial_{t} f+v \partial_{x} f=Q(f)
$$

where the source term $Q$, a nonlinear operator, describes the details of particle collisions.

The Euler equations can be recovered from (1) by taking moments $1, v, v^{2}$ and assuming that $f$ is a local Maxwellian distribution. The next member in the hierarchy is derived similarly but now with the moment vector

$$
m(v)=\left(1, v, v^{2}, v^{3}, v^{4}\right)^{T}
$$

and the distribution function

$$
f(x, v, t)=\exp \left(\alpha_{0}(x, t)+\alpha_{1}(x, t) v+\cdots+\alpha_{4}(x, t) v^{4}\right) .
$$

If we multiply the Boltzmann equation (1) with $m(v)$, integrate over $v$ and assume $f$ of the form (3) we obtain a system of equations for the coefficient vector $\alpha(x, t)$

$$
\partial_{t} \int_{\mathbb{R}} m f d x+\partial_{x} \int_{\mathbb{R}} v m f d v=\int_{\mathbb{R}} m Q(f) d v, \quad f=\exp \left(\alpha^{T} m\right) .
$$

Going over to the velocity moments as independent variables

$$
\rho(x, t)=\int_{\mathbb{R}} m(v) \exp \left(\alpha(x, t)^{T} m(v)\right) d v,
$$

we can write (4) in the form

$$
\partial_{t} \rho+\partial_{x} G(\rho)=r(\rho)
$$

where the flux vector $G$ is defined by

$$
G(\rho):=\int_{\mathbb{R}} v m(v) \exp \left(\alpha(\rho)^{T} m(v)\right) d v .
$$


Here we use the fact, that the coefficient vector $\alpha$ of an exponential density $\exp \left(\alpha^{T} m(v)\right)$ is in one-to-one correspondence with the vector of velocity moments $\rho[6]$. The right hand side $r(\rho)$ in (5) originates in the moment integral of the collision operator $Q(f)$ and is easily calculated if we use the BGK-type operator proposed in [6]

$$
\begin{gathered}
Q(f)=\frac{3}{2} \frac{n \theta}{\kappa(n, \theta)}\left(\frac{n}{\sqrt{2 \pi \theta}} \exp \left(-\frac{(v-u)^{2}}{2 \theta}\right)-f\right), \\
n=\int_{\mathbb{R}} f d v, \quad u=\frac{1}{n} \int_{\mathbb{R}} v f d v, \quad \theta=\frac{1}{n} \int_{\mathbb{R}}(v-u)^{2} f d v .
\end{gathered}
$$

Altogether, the one-dimensional Cauchy-problem for the five-moment system is abbreviated as

$$
\left\{\begin{array}{l}
\partial_{t} \rho+\partial_{x} G(\rho)=r(\rho), \\
\rho(x, 0)=\rho^{0}(x) \quad x \in \mathbb{R} .
\end{array}\right.
$$

For a complete understanding of the system it is inevitable to study the domain of definition of the flux vector $G$. From the definition (6) we see that $G(\rho)$ is well defined whenever a coefficient vector $\alpha$ can be determined such that

$$
\rho=\int_{\mathbb{R}} m(v) \exp \left(\alpha^{T} m(v)\right) d v
$$

Since we are interested only in integrable exponential densities, $\alpha$ is obviously restricted to the set

$$
\mathcal{A}=\left\{\left(\alpha_{0}, \ldots, \alpha_{4}\right): \alpha_{4}<0\right\} \cup\left\{\left(\alpha_{0}, \alpha_{1}, \alpha_{2}, 0,0\right): \alpha_{2}<0\right\} .
$$

Consequently, $G$ is defined on the range of moments of the exponential family

$$
\mathcal{R}=\left\{\int_{\mathbb{R}} m(v) \exp \left(\alpha^{T} m(v)\right) d v: \alpha \in \mathcal{A}\right\} .
$$

This set $\mathcal{R}$ also plays an important role in the constrained maximization problem of the entropy functional $H(f)=-\int_{\mathbb{R}} f \log f d v$

$$
\max \left\{H(f): f \geq 0, \int_{\mathbb{R}} m(v) f d v=\rho\right\} .
$$

Indeed, it can be shown that for $\rho \in \mathcal{R}$ the maximum in (10) is attained and has the form $\exp \left(\alpha^{T} m(v)\right)$ [10]. Conversely, if the maximum is attained, the vector $\rho$ must be contained in $\mathcal{R}$ as we will see below $([4,8]$ treat similar 
problems). According to [6], we obtain a natural entropy for system (8) if we consider (10) as a function of $\rho$. For $\rho \in \mathcal{R}$, the maximum can be expressed in terms of the unique coefficient vector $\alpha(\rho)$ corresponding to $\rho$ and by changing the sign we obtain the convex entropy

$$
\eta(\rho)=\alpha(\rho)^{T} \rho
$$

As far as the Cauchy-problem (8) is concerned, we have to make sure that $\rho^{0}(x) \in \mathcal{R}$ for all $x \in \mathbb{R}$, otherwise the problem is not well defined. If $\rho^{0}(\mathbb{R})$ is compactly contained in the interior of $\mathcal{R}$ and $\rho^{0}$ is sufficiently smooth (8) has locally a unique classical solution [7] since it is, in fact, a symmetric hyperbolic system [6]. According to [7] there are exactly two reasons for a breakdown of the classical solution in finite time. First, since (8) is a hyperbolic system, it is possible that the solution looses regularity. The second possibility is that the solution $\rho(x, t)$ escapes any compact subset $K \subset \mathcal{R}$ in finite time. In this context it is interesting to note that the production term $r(\rho)$ has the tendency to bring the system closer to the boundary of $\mathcal{R}$. This is due to the fact that the collision operator $Q$ relaxes the system to a state described by local Maxwellian distributions. Local Maxwellians, however, correspond to admissible $\alpha$-vectors with $\alpha_{4}=0$ which are located on $\partial \mathcal{A}$. Accordingly, the solution $\rho$ will tend to $\partial \mathcal{R}$. Whether the solution stays inside $\mathcal{R}$ during its evolution is a non trivial question whose answer again requires knowledge of the set $\mathcal{R}$.

In Section 2 we first show that the structure of $\mathcal{R}$ is essentially determined by its intersection with the affine space $\left\{\rho \in \mathbb{R}^{5}: \rho_{0}=1, \rho_{1}=0, \rho_{2}=1\right\}$. This reduced set is then characterized in Section 3. An estimate of $G(\rho)$ with $\rho$ close to $\partial \mathcal{R}$ is derived in Section 4. Then, the space homogeneous case is considered and some remarks on numerical aspects are given.

\section{Normalization}

In order to formulate results about the integrable exponential densities

$$
E=\left\{\exp \left(\alpha^{T} m\right): \alpha \in \mathcal{A}\right\}
$$

it is convenient to introduce the larger class

$$
D:=\left\{f \geq 0: v \mapsto\left(1+v^{4}\right) f(v) \in \mathbb{L}^{1}(\mathbb{R}), f \not \equiv 0\right\}
$$

which certainly contains $E$ as a subset. 
Given any nonnegative density $f \in D$ it is always possible to take out three degrees of freedom. Denoting the moment vector of $f$ by

$$
\mu(f)=\int_{\mathbb{R}} m(v) f(v) d v
$$

we define macroscopical density $n$, average velocity $u$ and temperature $\theta$ as

$$
n:=\mu_{0}, \quad u:=\frac{\mu_{1}}{\mu_{0}}, \quad \theta:=\frac{\mu_{2}}{\mu_{0}}-\left(\frac{\mu_{1}}{\mu_{0}}\right)^{2},
$$

where $\theta=\frac{1}{n} \int(v-u)^{2} f(v) d v$ is positive. Setting

$$
f^{*}(w):=\frac{\sqrt{\theta(f)}}{n(f)} f(\sqrt{\theta(f)} w+u(f))
$$

we have $\mu_{1}\left(f^{*}\right)=0$, and $\mu_{0}\left(f^{*}\right)=\mu_{2}\left(f^{*}\right)=1$ which shows that $f^{*}$ has lost three degrees of freedom compared to $f$ in the sense that the first three moments are constrained. The original density $f$ can be recovered from $f^{*}$ and $n, u, \theta$ by

$$
f(v)=\frac{n(f)}{\sqrt{\theta(f)}} f^{*}\left(\frac{v-u(f)}{\sqrt{\theta(f)}}\right) .
$$

On the level of moments, this means that the set of moments $\mu(D)$ is essentially characterized by the normalized moments $\mu\left(D^{*}\right)$. More precisely, $\mu(D)$ is easily recovered from $\mu\left(D^{*}\right)$ using the map

$$
\rho=\left(\begin{array}{c}
n \\
n u \\
n \theta+n u^{2} \\
n \theta^{\frac{3}{2}} \rho_{3}^{*}+3 n u \theta+n u^{3} \\
n \theta^{2} \rho_{4}^{*}+4 n \theta^{\frac{3}{2}} u \rho_{3}^{*}+6 n u^{2} \theta+n u^{4}
\end{array}\right)
$$

where $\rho^{*} \in \mu\left(D^{*}\right), u \in \mathbb{R}$ and $n, \theta \in \mathbb{R}^{+}$. It therefore suffices to understand the structure of the set $\mu\left(D^{*}\right)$ which is given by

Lemma 2.1 The set of normalized moments of nonnegative densities is

$$
\mu\left(D^{*}\right)=\left\{\left(1,0,1, \rho_{3}, \rho_{4}\right)^{T}: \rho_{4}>1+\rho_{3}^{2}\right\} .
$$

To any $\rho \in \mu\left(D^{*}\right)$ there exists a continuous, compactly supported density $f \in$ $D^{*}$ such that $\rho=\mu(f)$. 
Proof: For any nonzero polynomial $P(v)=c_{0}+c_{1} v+c_{2} v^{2}$ and any $f \in D^{*}$ with moments $\rho=\mu(f)$ we find

$$
0<\int_{\mathbb{R}} P^{2}(v) f(v) d v=\sum_{i, j=0}^{2} c_{i} c_{j} \int_{\mathbb{R}} v^{i+j} f(v) d v=\sum_{i, j=0}^{2} \rho_{i+j} c_{i} c_{j},
$$

with the Hankel matrix

$$
\left(\rho_{i+j}\right)=\left(\begin{array}{ccc}
1 & 0 & 1 \\
0 & 1 & \rho_{3} \\
1 & \rho_{3} & \rho_{4}
\end{array}\right)
$$

Due to (16) the matrix is positive definite so that

$$
0<\operatorname{det}\left(\rho_{i+j}\right)=\rho_{4}-\rho_{3}^{2}-1
$$

is a necessary condition on the moments in $\mu\left(D^{*}\right)$.

Conversely, if the moment condition $\rho_{4}>1+\rho_{3}^{2}$ is satisfied, then the sequence $\left(1,0,1, \rho_{3}, \rho_{4}\right)$ is a particular example of so called positive sequences [2]. A fundamental result in [1] states that to a positive sequence $\rho_{0}, \ldots, \rho_{4}$ there exists a positive measure

$$
\sigma=\sum_{i=1}^{3} \lambda_{i} \delta_{\xi_{i}}, \quad \lambda_{i}>0, \xi_{i} \in \mathbb{R}
$$

such that

$$
\rho=\int_{\mathbb{R}} m(v) d \sigma(v)
$$

In the following argument we show that $\sigma$ can even be replaced by a measure which has a density $f \in D^{*}$. Geometrically (18) means that the vector $\rho$ is contained in the interior of the conic hull of the vectors $m\left(\xi_{i}\right)$ since

$$
\rho=\sum_{i=1}^{3} \lambda_{i} \int_{\mathbb{R}} m(v) d \delta_{\xi_{i}}(v)=\sum_{i=1}^{3} \lambda_{i} m\left(\xi_{i}\right), \quad \lambda_{i}>0 .
$$

If we approximate the vectors $m\left(\xi_{i}\right)$ very accurately by vectors $l_{i}^{\epsilon}$ then $\rho$ will also be in the conic hull of $\left\{l_{i}^{\epsilon}: i=1,2,3\right\}$, i.e.

$$
\rho=\sum_{i=1}^{3} \eta_{i} l_{i}^{\epsilon}, \quad \eta_{i}>0
$$


Taking any symmetric, continuous, compactly supported function $\phi \in D^{*}$ we define $l_{i}^{\epsilon}$ as the moment vector of the scaled and shifted version

$$
\phi_{i}^{\epsilon}(v):=\frac{1}{\epsilon} \phi\left(\frac{v-\xi_{i}}{\epsilon}\right) .
$$

Then

$$
l_{i}^{\epsilon}-m\left(\xi_{i}\right)=\epsilon^{2}\left(\begin{array}{c}
0 \\
0 \\
1 \\
3 \xi_{i} \\
6 \xi_{i}^{2}+\kappa \epsilon^{2}
\end{array}\right)
$$

where $\kappa=\int v^{4} \phi(v) d v$. Hence, for $\epsilon$ small enough we can find positive parameters $\eta_{i}$ such that

$$
\rho=\sum_{i=1}^{3} \eta_{i} l_{i}^{\epsilon}=\sum_{i=1}^{3} \eta_{i} \int_{\mathbb{R}} m(v) \phi_{i}^{\epsilon}(v) d v=\int_{\mathbb{R}} m(v) f(v) d v
$$

with $f=\sum \eta_{i} \phi_{i}^{\epsilon} \in D^{*}$ which shows the sufficiency of the moment condition. By construction, $f$ is compactly supported and continuous.

Having gained complete knowledge about the set $\mu(D)$, the next step is to consider the subset $\mu(E)$ which contains the moments of exponential densities (the set $\mathcal{R}$ in our notation from Section 1). Before we turn to this problem in the next section, we give some remarks about the set $E$.

A nice property of the densities $f(v)=\exp \left(\alpha^{T} m(v)\right)$ is that they are form invariant under the $*-$ normalization, i.e. $E^{*} \subset E$. Indeed, (13) shows that

$$
\exp \left(\alpha^{T} m\right)^{*}(w)=\exp \left(\ln \frac{\sqrt{\theta}}{n}+\alpha^{T} m(\sqrt{\theta} w+u)\right)
$$

which is of the form $\exp \left(\alpha^{* T} m(v)\right)$ with $\alpha^{*}$ obtained by comparing coefficients in

$$
\alpha^{* T} m(w)=\ln \frac{\sqrt{\theta}}{n}+\alpha^{T} m(\sqrt{\theta} w+u) .
$$

Similarly, the reconstruction formula (14) can be expressed as a relation between $\alpha$-vectors.

Under the $*$-projection, the special exponential densities corresponding to $\alpha \in$ $\partial \mathcal{A}$ (the Maxwellians) turn into the single point

$$
\hat{f}(v)=\frac{1}{\sqrt{2 \pi}} e^{-\frac{1}{2} v^{2}}
$$


which is characterized by the vector $\hat{\alpha}=-\frac{1}{2}(\ln 2 \pi, 0,1,0,0)^{T}$ and the moments $\hat{\rho}=(1,0,1,0,3)^{T}$.

\section{Domain of definition}

In order to characterize the set $\mu(E)=\mathcal{R}$, or equivalently $\mu\left(E^{*}\right)$, we use its relationship to the maximum-entropy problem (10). The argument will be based on some general results which can be found for example in [10]. For convenience, we list these results here.

Instead of working with the entropy functional $H(f)=-\int f \ln f d v$ directly, we consider the so called relative entropy

$$
H(f, g):=\int_{\mathbb{R}} f \ln \frac{f}{g} d v .
$$

As reference density $g$ we choose the standard Maxwellian $\hat{f}$ defined in (19). Then

$$
H(f, \hat{f})=\frac{1}{2}\left(\mu_{0}(f) \ln (2 \pi)+\mu_{2}(f)\right)-H(f) .
$$

Under the constraint $\mu(f)=\rho$, maximizing $H(f)$ is therefore equivalent to minimizing $H(f, \hat{f})$.

We will use the following properties of the relative entropy.

Lemma 3.1 Let $f \in D$. Then

- $f \ln \frac{f}{\hat{f}} \geq-\frac{1}{e} \hat{f}$

- $H(f, \hat{f})$ is convex in $f$,

- if $f \in D^{*}$ then $H(f, \hat{f}) \geq 0$ with equality only in the case $f=\hat{f}$,

- if $f_{n} \rightarrow f$ in $\mathbb{L}^{1}(\mathbb{R})$ and $\left(f_{n}\right)_{n \in \mathbb{N}} \subset D^{*}$ then $H(f, \hat{f}) \leq \liminf _{n \rightarrow \infty} H\left(f_{n}, \hat{f}\right)$,

- if $F \subset D^{*}$ is convex, $\left(f_{n}\right)_{n \in \mathbb{N}} \subset F$ and $H\left(f_{n}, \hat{f}\right) \rightarrow \inf _{f \in F} H(f, \hat{f})<\infty$ then $f_{n} \rightarrow f$ in $\mathbb{L}^{1}(\mathbb{R})$.

Proof: Using the estimate $x \ln x \geq-\frac{1}{e}$ we get immediately

$$
f \ln \frac{f}{\hat{f}}=\hat{f}\left(\frac{f}{\hat{f}} \ln \frac{f}{\hat{f}}\right) \geq-\frac{1}{e} \hat{f} .
$$


This shows that the relative entropy is well defined since the negative part of $f \ln (f / \hat{f})$ is integrable. The convexity of $H(f, \hat{f})$ in the first argument follows directly from the corresponding property of $x \mapsto x \ln \frac{x}{y}$.

Since $f, \hat{f} \in D^{*}$ we have $\mu_{0}(f)=\mu_{0}(\hat{f})=1$ so that $f, \hat{f}$ are in fact probability densities. The remaining results can therefore be taken from Theorems 1.4.1, 1.5.5 and Theorem 3.1.1 in [10].

The second result we need for our argument concerns the solvability of the entropy optimization problems on general intervals in $v$ if the prescribed moment vector $\rho$ belongs to an exponential density.

Lemma 3.2 Let $I \subset \mathbb{R}$ be an interval and $\mathcal{A}^{I}$ the set of all $\alpha \in \mathbb{R}^{5}$ such that $\exp \left(\alpha^{T} m(v)\right)$ is integrable on I. Further, let

$$
D^{I}:=\left\{f \geq 0: v \mapsto\left(1+v^{4}\right) f(v) \in \mathbb{L}^{1}(I), f \not \equiv 0\right\}
$$

and

$$
E^{I}:=\left\{\exp \left(\alpha^{T} m\right): \alpha \in \mathcal{A}^{I}\right\} .
$$

The moments of $f \in D^{I}$ are denoted by

$$
\mu^{I}(f)=\int_{I} m(v) f(v) d v .
$$

Then the unique solution of the problem to minimize the relative entropy $H^{I}(f, \hat{f})=\int_{I} f \ln \frac{f}{\hat{f}} d v$ under the constraint $f \in D^{I}$ and $\mu^{I}(f)=\rho \in \mu^{I}\left(E^{I}\right)$ is given by

$$
f_{\text {opt }}(v)=\exp \left(\alpha^{T} m(v)\right)
$$

where $\alpha$ is determined uniquely by the relation $\mu^{I}\left(f_{\text {opt }}\right)=\rho$.

Proof: If we first restrict ourselves to the case $\rho_{0}=1$, Theorem 3.1.4 in [10] proves the result. The more general case $\rho_{0} \neq 1$ can easily be reduced to the previous situation by noting that with $\rho \in \mu^{I}\left(E^{I}\right)$ also $\frac{1}{\rho_{0}} \rho \in \mu^{I}\left(E^{I}\right)$ and that

$$
H^{I}\left(\rho_{0} f, \hat{f}\right)=\rho_{0} H^{I}(f, \hat{f})+\mu_{0}^{I}(f) \rho_{0} \ln \rho_{0} .
$$

With these tools at hand, we can now characterize the $\mu\left(E^{*}\right)$ as the set of those moment vectors for which the problem

$$
H(g, \hat{f})=\inf \{H(f, \hat{f}): f \in D, \mu(f)=\rho\}, \quad g \in D .
$$

is solvable. 
Lemma 3.3 Let $\rho \in \mu\left(D^{*}\right)$. Then the minimum in (21) is attained if and only if $\rho \in \mu\left(E^{*}\right)$.

Proof: First, we assume that $\rho \in \mu\left(E^{*}\right)$. Using Lemma 3.2 with $I=\mathbb{R}$ we see that (21) has a unique solution. Conversely, if (21) has a solution $g$, then in particular $g \not \equiv 0$ such that for some $n_{0} \in \mathbb{N}$

$$
g_{n}:=\left.g\right|_{(-n, n)} \not \equiv 0 \quad \forall n \geq n_{0} .
$$

On each interval $I_{n}=(-n, n)$ with $n \geq n_{0}$ the restriction $g_{n}$ then satisfies

$$
H\left(g_{n}, \hat{f}\right)=\inf \left\{H^{I_{n}}(f, \hat{f}): f \in D^{I_{n}}, \mu^{I_{n}}(f)=\mu^{I_{n}}\left(g_{n}\right)\right\}
$$

because otherwise one could decrease $H(g, \hat{f})$ by modifying $g$ on $I_{n}$. Granted that (22) is uniquely solved by a function of the form

$$
g_{n}(v)=\exp \left(\alpha_{n}^{T} m(v)\right),
$$

we can complete the proof of the lemma. Indeed, repeating the argument on $I_{n}$ with $g_{n+1}$ in place of $g$ we find $\left.g_{n+1}\right|_{I_{n}}=g_{n}$, respectively $\alpha_{n+1}=\alpha_{n}$ for all $n \geq n_{0}$. This yields $g(v)=\exp \left(\alpha_{n_{0}}{ }^{T} m(v)\right)$ so that $\rho \in \mu(E)$. The required solvability of problem $(22)$ is shown in the appendix.

Although (21) might not be solvable for every $\rho \in \mu(D)$, we can nevertheless define the entropy function

$$
h(\rho):=\inf \{H(f, \hat{f}): f \in D, \mu(f)=\rho\}, \quad \rho \in \mu(D) .
$$

To show that $h$ is well defined, we use relation (14) which yields

$$
H(f, \hat{f})=n H\left(f^{*}, \hat{f}\right)-n \ln \frac{n}{\sqrt{\theta}}-\frac{n}{2}\left(\theta-1+u^{2}\right) .
$$

Since $H\left(f^{*}, \hat{f}\right) \geq 0$ (Lemma 3.1), we immediately get $h(\rho)>-\infty$. On the other hand, there is at least one distribution function $f$ such that $H(f, \hat{f})$ is bounded so that also $h(\rho)<\infty$. Such an $f$, or more precisely its normalization $f^{*}$ is obtained with Lemma 2.1 due to the existence of a continuous, compactly supported solution $f^{*}$ of the moment problem.

We note that $h$ is closely related to the natural entropy $\eta$ of the hyperbolic system (8) which has been introduced in (11). Indeed, if $\rho$ is taken from the domain of definition $\rho \in \mathcal{R}=\mu(E)$ then Lemma 3.2 shows that

$$
h(\rho)=H\left(\exp \left(\alpha(\rho)^{T} m\right), \hat{f}\right)
$$


where $\alpha(\rho)$ is the unique coefficient vector $\alpha$ such that $\mu\left(\exp \left(\alpha(\rho)^{T} m\right)\right)=\rho$. Using (20) we get

$$
\begin{aligned}
h(\rho)=\frac{1}{2}\left(\rho_{0} \ln (2 \pi)+\rho_{2}\right)+\int_{\mathbb{R}} \alpha(\rho)^{T} m(v) \exp \left(\alpha(\rho)^{T} m(v)\right) d v & \\
= & \frac{1}{2}\left(\rho_{0} \ln (2 \pi)+\rho_{2}\right)+\eta(\rho)
\end{aligned}
$$

so that $\eta$ differs from $h$ only by a linear function in $\rho$. Since $\eta$ is $C^{\infty}$ smooth and strictly convex on $\mathcal{R}$ (see [6]) the same holds for $h$. In the following we need the important property that $h$ is monotonically decreasing in $\rho_{4}$. For $\rho \in \mathcal{R}$ this is easily calculated using the relation

$$
\rho_{j}=\left.\frac{\partial}{\partial \alpha_{j}} \mu_{0}\left(\exp \left(\alpha^{T} m\right)\right)\right|_{\alpha(\rho)} .
$$

Then

$$
\frac{\partial h(\rho)}{\partial \rho_{4}}=\frac{\partial \eta(\rho)}{\partial \rho_{4}}=\rho_{j} \frac{\partial \alpha_{j}(\rho)}{\partial \rho_{4}}+\alpha_{4}(\rho)=\frac{\partial}{\partial \rho_{4}}\left(\mu_{0}(\alpha(\rho))\right)+\alpha_{4}(\rho)=\alpha_{4}(\rho)<0 .
$$

The following Lemma shows that the two important properties of $h$ which are convexity and monotonicity in $\rho_{4}$ are valid for all moment vectors in $\mu\left(D^{*}\right)$.

Lemma 3.4 The entropy function $h$ is convex and on $\mu\left(D^{*}\right)$ it is decreasing in the last argument, i.e. for $\rho \in \mu\left(D^{*}\right)$ and $\delta \geq 0$

$$
h\left(\rho+\delta e_{4}\right) \leq h(\rho), \quad \text { where } e_{4}=(0,0,0,0,1)^{T} .
$$

In the interior of $\mu(E), h$ is even strictly convex and satisfies $\frac{\partial}{\partial \rho_{4}} h(\rho)<0$.

Proof: To check convexity of $h$ we simply use the definition (23). Given $\epsilon>0$, $\rho_{1}, \rho_{2} \in D$ and $\lambda \in[0,1]$ we can find densities $f_{1}, f_{2} \in D$ such that $\mu\left(f_{i}\right)=\rho_{i}$ and $H\left(f_{i}, \hat{f}\right) \leq h\left(\rho_{i}\right)+\epsilon$. Since $D$ is convex, $\lambda f_{1}+(1-\lambda) f_{2} \in D$ and hence

$$
\lambda \rho_{1}+(1-\lambda) \rho_{2}=\mu\left(\lambda f_{1}+(1-\lambda) f_{2}\right) \in \mu(D) .
$$

Using convexity of $H$ from Lemma 3.1, we conclude

$$
\begin{aligned}
& h\left(\lambda \rho_{1}+(1-\lambda) \rho_{2}\right) \leq H\left(\lambda f_{1}+(1-\lambda) f_{2}, \hat{f}\right) \\
& \quad \leq \lambda H\left(f_{1}, \hat{f}\right)+(1-\lambda) H\left(f_{2}, \hat{f}\right) \leq \lambda h\left(\rho_{1}\right)+(1-\lambda) h\left(\rho_{2}\right)+\epsilon .
\end{aligned}
$$

On the set $\mu(E)$ we have already seen that $h(\rho)$ is strictly convex and strictly decreasing in $\rho_{4}$. To show the monotonicity in the last argument for the general 
case, we again pick $\epsilon>0, \rho=\mu(f)$ with $f \in D^{*}$ and $H(f, \hat{f}) \leq h(\rho)+\epsilon$. For $\delta>0$, the moment vector $\rho+\delta e_{4}$ is contained in $D^{*}$ according to Lemma 2.1. A density $f_{r} \in D$, which approximately possesses this moment vector is given by

$$
f_{r}(v)=\left(1-\lambda_{r}\right) f(v)+\lambda_{r} g_{r}(v)
$$

with $g_{r}(v)=\delta \hat{f}(v-r)$ and $\lambda_{r}=1 / r^{4}$ sufficiently small. Indeed,

$$
\begin{aligned}
\rho_{r}=\mu\left(f_{r}\right) & =\left(1-\lambda_{r}\right) \mu(f)+\delta \lambda_{r} \int_{\mathbb{R}} m(v+r) \hat{f}(v) d v \\
& =\left(1-\frac{1}{r^{4}}\right) \rho+\delta e_{4}+\mathcal{O}\left(\frac{1}{r}\right) .
\end{aligned}
$$

Due to convexity, $h$ is continuous on $\mu(D)$ so that

$$
h\left(\rho_{r}\right) \longrightarrow h\left(\rho+\delta e_{4}\right) \quad \text { for } r \rightarrow \infty .
$$

On the other hand, we know that

$$
h\left(\rho_{r}\right) \leq H\left(f_{r}, \hat{f}\right) \leq\left(1-\lambda_{r}\right) H(f, \hat{f})+\lambda_{r} H\left(g_{r}, \hat{f}\right) .
$$

Since $H\left(g_{r}, \hat{f}\right)=\delta \ln \delta+\frac{\delta}{2} r^{2}$, we obtain $h\left(\rho_{r}\right) \leq h(\rho)+2 \epsilon$ for $r$ sufficiently large. Combining the two results we get

$$
h\left(\rho+\delta e_{4}\right) \leq h(\rho)
$$

which completes the proof of the lemma.

Finally, we are able to characterize the set $\mu\left(E^{*}\right)$ completely.

Theorem 3.5 The set $\mu\left(E^{*}\right)$ is given by

$$
\mu\left(E^{*}\right)=\mu\left(D^{*}\right) \backslash\left\{\left(1,0,1,0, \rho_{4}\right)^{T}: \rho_{4}>3\right\} .
$$

The entropy function $h$ is strictly convex on $\mu\left(E^{*}\right)$. Strict convexity is lost on the half line $\left\{\left(1,0,1,0, \rho_{4}\right)^{T}: \rho_{4} \geq 3\right\}$ where $h$ is equal to its absolute minimum $h(\hat{\rho})=0$.

Proof: Given any $\rho \in \mu\left(D^{*}\right)$ we define a non-empty convex set $F$ by

$$
F:=\left\{f \in D^{*}: \mu(f)=\rho\right\}
$$

Due to the definition of $h$ we can find a minimizing sequence $\left(f_{n}\right)_{n \in \mathbb{N}} \subset F$, i.e.

$$
H\left(f_{n}, \hat{f}\right) \longrightarrow h(\rho) \quad \text { for } n \rightarrow \infty .
$$


Using Lemma 3.1, we can infer the $\mathbb{L}^{1}$-convergence of $\left(f_{n}\right)$. The limit function $f$ is even contained in $D^{*}$. To see this, we first notice that with Fatou's lemma

$$
\int_{\mathbb{R}} v^{4} f(v) d v \leq \liminf _{n \rightarrow \infty} \int_{\mathbb{R}} v^{4} f_{n}(v) d v=\rho_{4}
$$

so that $f \in D$. On the other hand, the uniform bound on the fourth order moment yields convergence of all lower moments since for all $R>0$

$$
\int_{|v|>R}|v|^{i} f_{n}(v) d v \leq \int_{|v|>R}\left(\frac{|v|}{R}\right)^{4-i}|v|^{i} f_{n}(v) d v \leq R^{i-4} \rho_{4} .
$$

Using this estimate, we get with $\mathbb{L}^{1}$ convergence of $\left(f_{n}\right)$

$$
\int_{|v| \leq R} v^{i} f(v) d v=\lim _{n \rightarrow \infty} \int_{|v| \leq R} v^{i} f_{n}(v) d v=\rho_{i}+\mathcal{O}\left(\frac{1}{R}\right) \quad i=0, \ldots, 3
$$

which shows that

$$
\mu_{i}(f)=\rho_{i}, \quad i=0, \ldots, 3 \text { and } \mu_{4}(f) \leq \rho_{4} .
$$

From Lemma 3.4 we therefore get $h(\mu(f)) \geq h(\rho)$. Combined with the lower semi-continuity of the relative entropy from Lemma 3.1 this yields

$$
h(\rho) \leq h(\mu(f)) \leq H(f, \hat{f}) \leq \liminf _{n \rightarrow \infty} H\left(f_{n}, \hat{f}\right)=h(\rho) .
$$

In particular, $h(\mu(f))=H(f, \hat{f})$ which, with the help of Lemma 3.3, shows that $\mu(f) \in \mu\left(E^{*}\right)$. Invoking Lemma 3.2 with $I=\mathbb{R}$ we finally get

$$
f(v)=\exp \left(\alpha^{T} m(v)\right) \quad \text { with } \alpha \in \mathcal{A} .
$$

Consequently, the minimizing sequence always converges to an exponential density. However it is not yet clear whether the constraint $\mu(f)=\rho$ is satisfied. Due to (24) it can only happen that the fourth order moment drops, i.e.

$$
\mu(f)+\delta e_{4}=\rho, \quad \delta \geq 0 .
$$

To conclude the proof of the theorem, we distinguish two cases. First, we assume that the coefficient vector $\alpha$ of the limit density satisfies $\alpha_{4}=0$. In this case $f$ must be the normalized Maxwellian so that $\mu(f)=\hat{\rho}=(1,0,1,0,3)^{T}$. Consequently, the prescribed moment vector $\rho$ must be contained in the half line

$$
L:=\left\{\hat{\rho}+\delta e_{4}: \delta>0\right\}
$$


which starts in the point $\hat{\rho}$. At $\hat{\rho}$ one deduces from the definition of the relative entropy $h(\hat{\rho})=H(\hat{f}, \hat{f})=0$ which is the absolute minimum of $h$ on $\mu\left(D^{*}\right)$ since $H(g, \hat{f}) \geq 0$ for all $g \in D^{*}$ (Lemma 3.1). Combined with the monotonicity of $h$ we conclude

$$
h(\rho)=0 \quad \forall \rho \in L .
$$

In particular, if the constraint is based on $\rho \in L$ then the limit density $f=$ $\exp \left(\alpha^{T} m\right)$ satisfies $0=h(\rho)=h(\mu(f))=H(f, \hat{f})$ which gives $f=\hat{f}$ (Lemma 3.1) and hence

$$
\mu_{4}(f)=\mu_{4}(\hat{f})=3<\rho_{4},
$$

i.e. the highest component of the moment vector really drops in the limit.

In the remaining case $\alpha_{4}<0$, we know from Lemma 3.4 that $h$ is locally strictly decreasing in the last argument. Together with the overall monotonicity this yields $h(\rho)=h\left(\mu(f)+\delta e_{4}\right)<h(\mu(f))$ if $\delta>0$ in contradiction to (25). Consequently, $\delta=0$ so that $f$ satisfies the constraint $\mu(f)=\rho$ and thus is a solution of the optimization problem. Altogether we find that only for $\rho \in L$ the limit of the minimizing sequence does not satisfy the moment condition $\mu(f)=\rho$ so that with Lemma 3.3

$$
\mu\left(E^{*}\right)=\mu\left(D^{*}\right) \backslash L
$$

A graphical representation of the set $\mu\left(E^{*}\right)$ in the $\left(\rho_{3}, \rho_{4}\right)$ plane is given in Figure 1.

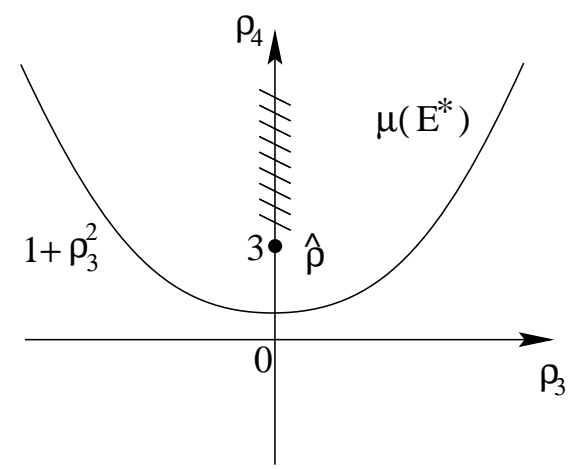

Figure 1: Structure of $\mu\left(E^{*}\right)$

Finally, let us summarize the implications of Theorem 3.5 on the moment system (8). Since $\mu\left(E^{*}\right)$ is the intersection of the domain of definition $\mathcal{R}$ with the affine subspace

$$
\left\{\left(1,0,1, \rho_{3}, \rho_{4}\right)^{T}: \rho_{3}, \rho_{4} \in \mathbb{R}\right\}
$$


the non convexity of $\mu\left(E^{*}\right)$ immediately implies non convexity of $\mathcal{R}$. Nevertheless, $\mu\left(E^{*}\right)$ is still star shaped with respect to the Maxwellian point $\hat{\rho}$. In Section 5 we will see that this is enough to guarantee that the solution of the space homogeneous problem stays inside $\mathcal{R}$ for all times.

The second conclusion we draw from Theorem 3.5 is that the continuous extension of the natural entropy $\eta$ to the set $\mu(D)$ of all moments of non negative densities it is no longer strictly convex. In the next section we show that a corresponding extension of the flux function $G$ is not possible since it is singular on the excluded half line so that the gap in the domain of definition cannot be closed by extension. The singularity of the flux function also has important consequences on numerical simulations because evaluating the flux for states close to the half line is extremely hard. In test cases like Riemann problems, however, the solution develops exactly in this numerically dangerous area because the constant left and right states are typically equilibrium states (i.e. Maxwellian states). A few comments on numerical problems are given in Section 6 .

We conclude with the remark that the considerations presented here are also applicable to higher dimensional cases like the 14 moment system. The main argument is again that the entropy function $h$ has its minimum in Maxwellian states and that it decreases in the last argument. Then, automatically, it must be constant on half lines starting in Maxwellian states with the same consequences as described above.

\section{A flux estimate}

Recalling the definition of the flux vector $G$

$$
G(\rho)=\int_{\mathbb{R}} v m(v) \exp \left(\alpha(\rho)^{T} m(v)\right) d v .
$$

we see that only the last component of $G$ is non trivial. Indeed, $G(\rho)=$ $\left(\rho_{1}, \rho_{2}, \rho_{3}, \rho_{4}, G_{4}(\rho)\right)^{T}$ with

$$
G_{4}(\rho)=\int_{\mathbb{R}} v^{5} \exp \left(\alpha(\rho)^{T} m(v)\right) d v
$$

For this velocity moment of order five it is possible to derive the following estimate.

Lemma 4.1 Let $\rho=\rho(\alpha) \in \mu\left(E^{*}\right)$. Then

$$
\rho_{3} G_{4}(\rho) \geq\left(\rho_{4}-3\right)\left(\rho_{4}-1\right)+4 \rho_{3}^{2}
$$


where equality appears only for the standard Maxwellian with $\rho=\hat{\rho}$.

Proof: We start with the relation

$$
\begin{aligned}
0 & =\int_{\mathbb{R}} \frac{d}{d v}\left[v^{k} \exp \left(\alpha^{T} m(v)\right)\right] d v \\
& =\int_{\mathbb{R}}\left(k v^{k-1}+v^{k} \alpha^{T} m^{\prime}(v)\right) \exp \left(\alpha^{T} m(v)\right) d v \\
& =4 \alpha_{4} \rho_{k+3}+3 \alpha_{3} \rho_{k+2}+2 \alpha_{2} \rho_{k+1}+\alpha_{1} \rho_{k}+k \rho_{k-1},
\end{aligned}
$$

which holds for any $k \in \mathbb{N}_{0}$ (the $v^{k}$-moment is denoted $\rho_{k}$ ). In the following we will concentrate on the equations $k=0, \ldots, 3$ which can be written in the form

$$
\left(\begin{array}{llll}
\rho_{0} & 2 \rho_{1} & 3 \rho_{2} & 4 \rho_{3} \\
\rho_{1} & 2 \rho_{2} & 3 \rho_{3} & 4 \rho_{4} \\
\rho_{2} & 2 \rho_{3} & 3 \rho_{4} & 4 \rho_{5} \\
\rho_{3} & 2 \rho_{4} & 3 \rho_{5} & 4 \rho_{6}
\end{array}\right)\left(\begin{array}{l}
\alpha_{1} \\
\alpha_{2} \\
\alpha_{3} \\
\alpha_{4}
\end{array}\right)=\left(\begin{array}{c}
0 \\
-\rho_{0} \\
-2 \rho_{1} \\
-3 \rho_{2}
\end{array}\right)
$$

or introducing

$$
\begin{aligned}
\bar{\alpha} & =\left(\alpha_{1}, \ldots, \alpha_{4}\right)^{T}, \quad h=-\left(0, \rho_{0}, 2 \rho_{1}, 3 \rho_{2}\right)^{T}, \\
D & =\operatorname{diag}(1,2,3,4), \quad M_{i j}=\rho_{i+j}, \quad i, j=0, \ldots, 3,
\end{aligned}
$$

as

$$
M D \bar{\alpha}=h .
$$

First we note that the symmetric matrix $M$ is positive definite, since

$$
\boldsymbol{\beta}^{T} M \boldsymbol{\beta}=\int_{\mathbb{R}}\left(\sum_{i=0}^{3} \beta_{i} v^{i}\right)^{2} \exp \left(\alpha^{T} m(v)\right) d v>0 \quad \text { if } \quad \boldsymbol{\beta} \neq 0 .
$$

Consequently, $M D$ is invertible so that $\bar{\alpha}=(M D)^{-1} h$. If $\alpha$ is in the interior of $\mathcal{A}$, we know that

$$
0>\alpha_{4}=e_{4}^{T}(M D)^{-1} h=\frac{1}{4}\left(M^{-1} e_{4}\right)^{T} h,
$$

with $e_{4}=(0,0,0,1)^{T}$. In order to simplify this inequality further, we write $M$ as a block matrix

$$
M=\left(\begin{array}{cc}
A & g \\
g^{T} & \gamma
\end{array}\right), \quad A \in \mathbb{R}^{3,3}, \quad g \in \mathbb{R}^{3}, \quad \gamma \in \mathbb{R} .
$$

Obviously

$$
M\left(\begin{array}{c}
-A^{-1} g \\
1
\end{array}\right)=\left(\gamma-g^{T} A^{-1} g\right) e_{4}
$$


and $\gamma-g^{T} A^{-1} g \neq 0$ since $M$ is regular. Hence

$$
M^{-1} e_{4}=\frac{1}{\gamma-g^{T} A^{-1} g}\left(\begin{array}{c}
-A^{-1} g \\
1
\end{array}\right)
$$

In particular

$$
\frac{1}{\gamma-g^{T} A^{-1} g}=e_{4}^{T} M^{-1} e_{4}>0
$$

because $M^{-1}$ is the inverse of a positive definite matrix. Condition (26) is therefore equivalent to

$$
\left(-g^{T} A^{-1}, 1\right) h<0 .
$$

Now we use that $\rho$ is normalized to write

$$
A=\left(\begin{array}{ccc}
1 & 0 & 1 \\
0 & 1 & \rho_{3} \\
1 & \rho_{3} & \rho_{4}
\end{array}\right)
$$

which gives

$$
-A^{-1}=\frac{1}{\rho_{4}-\rho_{3}^{2}-1}\left(\begin{array}{ccc}
\rho_{3}^{2}-\rho_{4} & -\rho_{3} & 1 \\
-\rho_{3} & 1-\rho_{4} & \rho_{3} \\
1 & \rho_{3} & 1
\end{array}\right) .
$$

For $\rho \in \mathcal{R}^{*}$ we have $\rho_{4}-\rho_{3}^{2}-1>0$ so that $(27)$ with $g=\left(\rho_{3}, \rho_{4}, \rho_{5}\right)^{T}$ and $h=(0,-1,0,-3)^{T}$ transforms into

$$
\rho_{3}^{2}+\rho_{4}\left(\rho_{4}-1\right)-\rho_{3} \rho_{5}-3\left(\rho_{4}-\rho_{3}^{2}-1\right)<0 .
$$

Replacing $\rho_{5}=G_{4}(\rho)$ and rearranging terms leads to the desired inequality

$$
\rho_{3} G_{4}(\rho)>\left(\rho_{4}-3\right)\left(\rho_{4}-1\right)+4 \rho_{3}^{2} .
$$

Inserting the special case $\rho=\hat{\rho}=(1,0,1,0,3)^{T}$ we find equality.

In the case $\rho_{3}=0$ the Lemma yields the known condition $\left(3-\rho_{4}\right)\left(\rho_{4}-1\right) \geq 0$. On the one hand, $\rho_{4}$ has to be less than three since otherwise the moment vector is located on the forbidden half-line in Figure 1. The requirement $\rho_{4}>1$ reflects the condition due to the parabolic boundary of $\mu\left(D^{*}\right)$.

A new result is obtained if we choose for example $\rho_{3}>0$ and $\rho_{4} \geq 3+\epsilon$ with $\epsilon>0$ fixed. Then the inequality yields

$$
G_{4}(\rho) \geq \frac{\epsilon(2+\epsilon)}{\rho_{3}}+4 \rho_{3} \rightarrow+\infty \quad \text { for } \rho_{3} \searrow 0 .
$$


Similarly, we get for $\rho_{3}<0$ and $\rho_{4}$ as above

$$
G_{4}(\rho) \leq \frac{\epsilon(2+\epsilon)}{\rho_{3}}+4 \rho_{3} \rightarrow-\infty \quad \text { for } \rho_{3} \nearrow 0 .
$$

Whether this singular behavior of the flux results in the tendency of the system to draw away from the boundary is an interesting question whose answer is not at all obvious.

A final remark concerns the relation between the system (8) and the symmetric hyperbolic systems of gas dynamics considered in the theory of extended thermodynamics [9]. It has been shown [3] that the systems of extended thermodynamics can be derived by expanding systems which are obtained from a maximum entropy approach like the one of Levermore. This expansion is carried out in the neighborhood of equilibrium, i.e. around a Maxwellian state. However, the resulting flux function neither has singularities nor is there any restriction in the domain of definition locally around equilibrium. This shows that in this approximation process important features of the original system are lost. For initial values which are close to equilibrium but also close to the excluded half line we can thus expect a noticeable difference in the behavior of the maximum entropy system versus the extended thermodynamics one.

\section{The space homogeneous case}

In the space homogeneous case equation (8) turns into a system of ODEs with the constant state $\rho^{0} \in \mathcal{R}$ as initial value

$$
\frac{d \rho}{d t}=r(\rho), \quad \rho(0)=\rho^{0} .
$$

The function $r$ on the right hand side is obtained by integrating the BGKoperator (7) after multiplying with the vector of monomials $m(v)$. We get

$$
r(\rho)=\frac{3 n \theta}{2 \kappa(n, \theta)}\left(0,0,0,-q, 3 n \theta^{2}-s-4 q u\right)^{T}
$$

with $n, u, \theta, q, s$ depending on $\rho$ according to

$$
\left(\begin{array}{l}
n \\
u \\
\theta \\
q \\
s
\end{array}\right)=\left(\begin{array}{c}
\rho_{0} \\
\rho_{1} / n \\
\rho_{2} / n-u^{2} \\
\rho_{3}-3 n \theta u-n u^{3} \\
\rho_{4}-4 q u-6 n \theta u^{2}-n u^{4}
\end{array}\right) .
$$


Conservation of the first three moments (mass, momentum and energy) is reflected by $r_{0}=r_{1}=r_{2}=0$. Only for the third and fourth moment, equation (28) is nontrivial. Using the relation between $q, s$ and $\rho$ we obtain

$$
\begin{aligned}
& \dot{\rho}_{3}=-\lambda \rho_{3}+\lambda\left(n u^{3}+3 n \theta u\right), \\
& \dot{\rho}_{4}=-\lambda \rho_{4}+\lambda\left(n u^{4}+6 n \theta u^{2}+3 n \theta^{2}\right),
\end{aligned}
$$

with the abbreviation

$$
\lambda=\frac{3 n \theta}{2 \kappa(n, \theta)} .
$$

We remark that $n, u, \theta$ do not depend on time because they are calculated from the constant moments $\rho_{0}, \rho_{1}, \rho_{2}$. The solution of the decoupled linear system is easily found to be

$$
\begin{aligned}
& \rho_{3}(t)=\rho_{3}(0) e^{-\lambda t}+\left(n u^{3}+3 n \theta u\right)\left(1-e^{-\lambda t}\right), \\
& \rho_{4}(t)=\rho_{4}(0) e^{-\lambda t}+\left(n u^{4}+6 n \theta u^{2}+3 n \theta^{2}\right)\left(1-e^{-\lambda t}\right) .
\end{aligned}
$$

To check whether the solution stays inside $\mathcal{R}$ we consider the corresponding normalized moment vector. Inverting relation (15), we first obtain

$$
\rho^{*}=\left(\begin{array}{c}
1 \\
0 \\
1 \\
n^{-1} \theta^{-\frac{3}{2}}\left(\rho_{3}-3 n u \theta-n u^{3}\right) \\
n^{-1} \theta^{-2}\left(\rho_{4}-4 \rho_{3} u+6 n u^{2} \theta+3 n u^{4}\right)
\end{array}\right) .
$$

Plugging in the solution of the space homogeneous problem, we find after some algebra

$$
\begin{aligned}
& \rho_{3}^{*}(t)=\rho_{3}^{*}(0) e^{-\lambda t} \\
& \rho_{4}^{*}(t)=\rho_{4}^{*}(0) e^{-\lambda t}+3\left(1-e^{-\lambda t}\right) .
\end{aligned}
$$

Hence, the graph of the solution in the $\mu\left(E^{*}\right)$ plane is just a straight line connecting the normalized initial state $\rho^{*}(0)$ with the Maxwellian state $\hat{\rho}=$ $(1,0,1,0,3)^{T}$ which is reached at $t=\infty$. Since $\mu\left(E^{*}\right)$ is star shaped with respect to $\hat{\rho}$ the solution stays inside the domain of definition for all times.

If we choose $\rho(0)$ appropriately it can obviously be achieved that the solution stays arbitrarily close to the boundary

$$
L:=\left\{\hat{\rho}+\delta e_{4}: \delta>0\right\} .
$$

Whether a slight perturbation of this homogeneous situation causes the solution to leave the domain of definition or not is of course an interesting question. Unfortunately, numerical experiments which could shed some light on the problem are extremely difficult in that case as explained in the following section. 


\section{$6 \quad$ Numerical aspects}

To solve the moment system (8), any numerical method needs to evaluate the flux

$$
G(\rho)=\int_{\mathbb{R}} v m(v) \exp \left(\alpha(\rho)^{T} m(v)\right) d v .
$$

Assuming that $\alpha(\rho)$ is known with $\alpha_{4}<0$, the integral cannot be calculated analytically so one has to resort to numerical quadrature rules.

A tempting idea is to use Gauss-Hermite integration of sufficiently high order because this method reproduces the moments of a normalized Maxwellian exactly. With a good guess of the first three moments (e.g. the values from a previous time step) we can approximately normalize $\exp \left(\alpha^{T} m(v)\right)$ and if we are close to the normalized Maxwellian we would expect precise results also in that case. However, the idea has two disadvantages. First, with Gauss integration the quality of the results is difficult to estimate and second, the method cannot work for all vectors $\alpha$. To calculate for example $\int v^{4} \exp \left(\alpha^{T} m(v)\right) d v$ with $\alpha=(0.00045,-0.0425,-1.001,0.02832,-0.000201)^{T}$ the function depicted in Figure 2 has to be integrated.

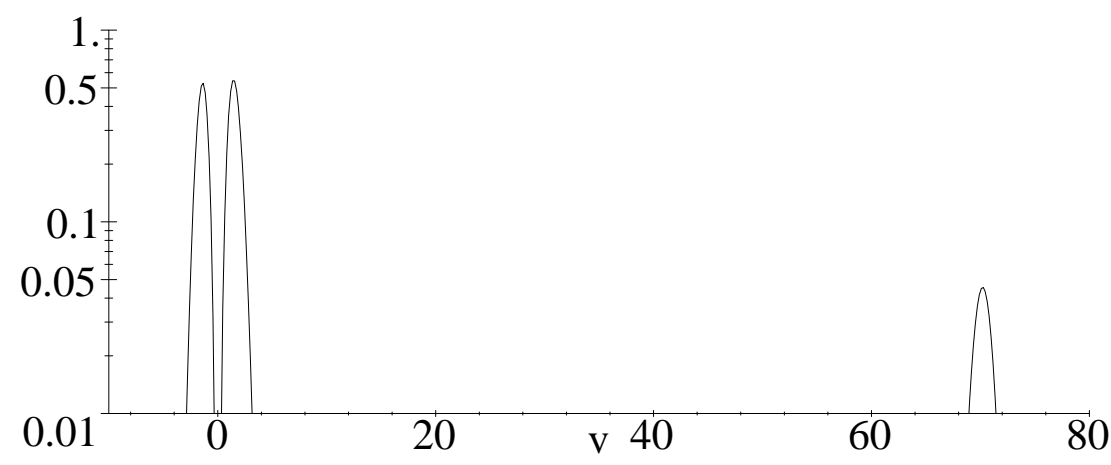

Figure 2: An example of $v^{4} \exp \left(\alpha^{T} m(v)\right)$

Gauss-Hermite integration even of order 20, however, uses information of $f$ only in the interval $[-5.5,5.5]$ so that the contribution at $v \approx 70$ cannot be captured. To explain the underlying principle in the example we consider the polynomial

$$
P(v)=\alpha^{T} m(v)=\alpha_{0}+\alpha_{1} v+\alpha_{2} v^{2}+\alpha_{3} v^{3}+\alpha_{4} v^{4} .
$$

Since $\alpha_{4}<0, P$ and hence also $\exp (P)$ can have at most two maxima. If $\alpha_{3} /\left|\alpha_{4}\right|$ is large, one of these maxima can be located at $v \gg 1$. Indeed, for

$$
P^{\prime}(v)=v^{2}\left(\frac{\alpha_{1}}{v^{2}}+\frac{2 \alpha_{2}}{v}+3 \alpha_{3}+4 \alpha_{4} v\right)
$$


to vanish at large $v, 3 \alpha_{3}+4 \alpha_{4} v$ has to be close to zero which yields $v \approx \frac{3 \alpha_{3}}{4\left|\alpha_{4}\right|}$. An additional complication comes from the fact that height and position of such maxima are extremely sensitive to the parameters $\alpha$. For the calculation of a moment $v^{k}$ this is disadvantageous in so far as error in the height of the maximum is amplified by the large factor $v^{k}$.

In the set $\mu\left(E^{*}\right)$ (see Figure 1 ) moment vectors corresponding to large $\alpha_{3} /\left|\alpha_{4}\right|$ ratios are situated around the excluded half-line. This in not surprising if we reconsider the results of Section 4 . We have seen that the fifth order moment explodes if $\rho$ approaches the half line while $\rho_{4}$ stays bounded. Such a behavior can be explained by a maximum in the corresponding exponential densities which moves to infinity and is scaled in such a way that its contribution to the fourth order moment $\rho_{4}$ is always $\mathcal{O}(1)$. For all higher moments, the contribution due to this maximum then obviously grows without bound. If Gauss-Hermite integration is used to evaluate the flux function (as for example in [5] for the 14 moment case) these contributions at high velocities are automatically suppressed. Consequently, the approximated flux function cannot show a singular behavior. This is similar to the approach in extended thermodynamics which we have discussed in Section 4. Here, the original exponential density is replaced by a polynomial perturbation of the Maxwellian. Due to the slow growth of polynomials at infinity compared to the exponential decay of the Maxwellian these densities also do not show noticeable contributions at high velocities so that the resulting flux function does not show a singular behavior.

Beside integrating moments of exponential densities, the evaluation of the function $\alpha(\rho)$ is a crucial step in the calculation of the flux $G(\rho)$. The guideline for this is already given in [6]. It turns out, that $\alpha(\rho)$ is the unique minimizer of the problem

$$
\min _{\alpha \in \mathcal{A}} z(\alpha), \quad z(\alpha)=\int_{\mathbb{R}} \exp \left(\alpha^{T} m(v)\right) d v-\alpha^{T} \rho .
$$

At this point, we just want to state a few properties of (30). It is a constrained minimization problem with the set constraint $\alpha \in \mathcal{A} \subset \mathbb{R}^{5}$. Since the objective function $z$ cannot be evaluated in the complement of $\mathcal{A}$ we have to be careful in selecting search directions and doing line search in the vicinity of $\partial \mathcal{A}$. Up to the inherent problems described above, calculating arbitrary derivatives of $z$ is of the same complexity as evaluating the objective function itself. We have

$$
\begin{aligned}
\nabla_{\alpha} z(\alpha) & =\int_{\mathbb{R}} m(v) \exp \left(\alpha^{T} m(v)\right) d v-\rho, \\
\mathcal{H}[z](\alpha) & =\int_{\mathbb{R}} m(v) \otimes m(v) \exp \left(\alpha^{T} m(v)\right) d v .
\end{aligned}
$$


The condition number of the Hessian matrix $\mathcal{H}$ is an indicator for the expected degree of difficulty of problem (30) since it determines the speed of convergence of the fundamental steepest descent method. For the standard Maxwellian $\hat{\alpha}=$ $(-\ln \sqrt{2 \pi}, 0,-1 / 2,0,0)^{T}$ the condition number is already 344 but the situation can be much worse as the example $\alpha=(-0.9,-0.175,-0.526,0.0594,-0.0017799)^{T}$ shows, where the condition number is larger than $2 \cdot 10^{5}$. Again, this is not surprising since the lowest diagonal element in the Hessian is the moment $v^{8}$. In a situation like in Figure 2 this entry will be very large compared to the highest diagonal element which is $\rho_{0}=1$ in the normalized case. Since the condition number can be estimated by the ratio between the largest and the smallest diagonal entry we see that numerical problems in the calculation of $\alpha(\rho)$ also occur near the excluded half-line in the set $\mu\left(E^{*}\right)$.

\section{Conclusions}

We have analyzed the structure of the domain of definition of Levermore's five moment system which can be viewed as a model problem for the full dimensional case. The method is based on a detailed investigation of the solvability of a corresponding maximum entropy problem. It turns out that there are admissible moment constraints for which this maximum entropy problem is not solvable. These exceptional moment vectors are characterized as the set where the entropy function connected with Levermore's hyperbolic system looses strict hyperbolicity and where the flux function becomes singular.

This interesting behavior is not captured if the flux function is approximated by certain quadrature rules or if an expansion around equilibrium is applied as in the theory of extended thermodynamics. The solution of the space homogeneous problem has been shown to stay in the domain of definition but what happens in the non homogeneous case remains an open problem.

\section{Appendix}

Our aim is to show that the minimum

$$
\min \left\{H^{I}(f, \hat{f}): f \in D^{I}, \mu^{I}(f)=\rho\right\}
$$

is attained whenever $I$ is a bounded interval and $\rho \in \mu^{I}\left(D^{I}\right)$ (we use the notation from Lemma 3.2). For the case $I=[0,1]$ this result has been proved in [8] with a different technique. Since the statement holds if $\rho \in \mu^{I}\left(E^{I}\right)$ (see Lemma 3.2) it suffices to show $\mu^{I}\left(E^{I}\right)=\mu^{I}\left(D^{I}\right)$. 
For a fixed $\rho \in \mu^{I}\left(D^{I}\right)$ we introduce the $C^{\infty}$-function

$$
z^{I}(\alpha)=\int_{I} \exp \left(\alpha^{T} m(v)\right) d v-\alpha^{T} \rho, \quad \alpha \in \mathbb{R}^{5}
$$

which is strictly convex because of positive definiteness of the Hessian

$$
\mathcal{H}\left[z^{I}\right](\alpha)=\int_{I} m(v) \otimes m(v) \exp \left(\alpha^{T} m(v)\right) d v .
$$

In order to make sure that $z^{I}$ does not attain its minimum at the boundary, which is at $|\alpha|=\infty$ in this case, we will show

$$
\lim _{s \rightarrow \infty} z^{I}(s \alpha)=+\infty \quad \forall \alpha \in \mathbb{R}^{5} \backslash\{0\} .
$$

Being sure that the minimum is attained for some finite $\bar{\alpha} \in \mathbb{R}^{5}$ we get from the necessary condition $\nabla z^{I}(\bar{\alpha})=0$

$$
\int_{I} m(v) \exp \left(\bar{\alpha}^{T} m(v)\right) d v-\rho=0
$$

which means $\rho \in \mu^{I}\left(E^{I}\right)$. Since obviously $\mu^{I}\left(E^{I}\right) \subset \mu^{I}\left(D^{I}\right)$ we thus proved the

Lemma Let I be a bounded interval. Then $\mu^{I}\left(E^{I}\right)=\mu^{I}\left(D^{I}\right)$ which means that the minimum in (31) is always attained.

All what remains to show is (32). For a given $0 \neq \alpha \in \mathbb{R}^{5}$ we distinguish two cases: First we assume $\alpha^{T} m(v) \leq 0$ for all $v \in I$. Since $\rho \in \mu^{I}\left(D^{I}\right)$ we can write $\rho=\mu^{I}(f)$ with $f \in D^{I}$ and, due to the definition of $D^{I},\{v \in I: f(v)>0\}$ has positive measure in $I$. Hence also

$$
\Omega:=\left\{v \in I: \alpha^{T} m(v)<0, f(v)>0\right\}
$$

has positive measure because $\left\{v: \alpha^{T} m(v)=0\right\}$ has measure zero by the exclusion of $\alpha=0$. Consequently,

$$
\alpha^{T} \rho=\alpha^{T} \mu^{I}(f)=\int_{\Omega} \alpha^{T} m(v) \exp \left(\alpha^{T} m(v)\right) d v<0
$$

which gives (32) because $z^{I}(s \alpha)>-s \alpha^{T} \rho$.

In the second case we can assume the existence of some $v_{0} \in I$ for which $\alpha^{T} m\left(v_{0}\right)>0$. Then, also on a small neighborhood $B \subset I$ of $v_{0}$ we can obtain $\alpha^{T} m(v) \geq \epsilon>0$ for all $v \in B$ so that

$$
z^{I}(s \alpha) \geq \operatorname{vol}(B) \exp (s \epsilon)-s \alpha^{T} \rho
$$

which also gives (32). 


\section{References}

[1] N. I. Akhiezer, M. Krein, Some Questions in the Theory of Moments, Transl. of Math. Monographs, Vol. 2, 1962

[2] N. I. AKHIEZER, The classical moment problem and some related questions in analysis, Oliver \& Boyd, 1965

[3] W. Dreyer, Maximisation of the entropy in non-equilibrium, J. Phys. A: Math. Gen., 20, 6505-6517, 1987

[4] A. Kociszewski, The existence conditions for maximum entropy distributions, having prescribed the first three moments, J. Phys. A: Math. Gen., 19, L823-L827, 1986

[5] P. Le Tallec, J. P. Perlat, Numerical analysis of Levermore's moment system, Rapport de recherche No. 3124, INRIA Rocquencourt, 1997

[6] C. D. Levermore, Moment Closure Hierarchies for Kinetic Theories, J. Stat. Phys., 83, 1021-1065, 1996

[7] A. MAJDA, Compressible fluid flow and systems of conservation laws in several space variables, Springer, 1984

[8] L. R. Mead, N. Papanicolaou, Maximum entropy in the problem of moments, J. Math. Phys., 25, 2404-2417, 1984

[9] I. Müller, T. Ruggeri, Extended Thermodynamics, Springer Verlag New York, 1993

[10] S. Ihara, Information Theory for Continuous Systems, World Scientific, 1993 